OPEN ACCESS

Citation: E. Pelizzari (2021) La donna in Africa e il suo ruolo educativo in seno alla famiglia: un possibile baluardo contro la radicalizzazione dei più giovani? II caso della regione sahelo-sahariana.Rief 18, 2 : pp. 17-25. doi: https://doi.org/ 10.36253/rief-10482.

Copyright: (c) 2021 E. Pelizzari. This is an open access, peer-reviewed article published by Firenze University Press (https:// oaj.fupress.net/index.php/rief) and distributed under the terms of the Creative Commons Attribution License, which permits unrestricted use, distribution, and reproduction in any medium, provided the original author and source are credited.

Data Availability Statement: All relevant data are within the paper and its Supporting Information files.

Competing Interests: The Author(s) declare(s) no conflict of interest.

\section{La donna in Africa e il suo ruolo educativo in seno alla famiglia: un possibile baluardo contro la radicalizzazione dei più giovani? Il caso della regione sahelo-sahariana}

\author{
Elisa Pelizzari ${ }^{I}$
}

\begin{abstract}
A partire dal rapporto, coniugato al femminile, "educazione familiare-prevenzione del radicalismo giovanile" e basandomi sulle ricerche che conduco da una decina d'anni in Paesi quali il Mali, il Senegal e la Repubblica di Guinea, intendo procedere con un discorso a carattere antropologico con l'obiettivo di offrire qualche pista di confronto per chi opera, da noi, a livello pedagogico, in seno a realtà marcate dal meticciato culturale e dall'immigrazione.
\end{abstract}

Parole chiave: donne, educazione, radicalismo, Africa, immigration.

\section{Abstract}

Starting from the relationship, conjugated to the feminine, "family education-prevention of youth radicalism" and based on the research I have been conducting for ten years in countries such as Mali, Senegal and the Republic of Guinea, I intend to proceed with a speech of an anthropological nature with the aim of offering some paths of comparison for those who work at a pedagogical level, within realities marked by cultural hybridization and immigration.

Keywords: women, education, radicalisation, Africa, immigration.

${ }^{1}$ Ph.D. in Antropologia sociale ed Etnologia, specialità Africa subsahariana (EHESS - Paris); attuale Responsabile della casa editrice L'Harmattan Italia. 


\section{Premessa metodologica}

Questo articolo nasce da un intervento che ho tenuto in occasione del convegno organizzato il 7 novembre 2020 presso il Dipartimento di Formazione, Lingue, Intercultura, Letterature e Psicologia (FORLILPSI) dell'Università degli Studi di Firenze, dedicato al tema del ruolo del femminile nel prevenire, oggi, in ambito familiare, comportamenti antisociali o forme di radicalizzazione violenta a matrice religiosa, etnica, culturale, politica ecc.

Il riferimento a un contesto dai risvolti più intimi, quale la famiglia, permette meglio di altri di porre l'attenzione sulla funzione cruciale che possono svolgere le donne nello sviluppo, presso le nuove generazioni, di atteggiamenti costruttivi, aperti al dialogo, lontani dagli estremismi, tanto nelle aree appartenenti al cosiddetto Occidente (Biagioli, Gonzáles-Monteagudo et al., 2018; Silva, 2017, 2004), quanto in parti del mondo lontane da noi per costumi e tradizioni, come le regioni sahelo-sahariane dell'Africa, oggetto del presente lavoro.

Indipendentemente dal contesto geografico, in effetti, chiave del dibattito legato alla salvaguardia da chiusure settarie e fanatiche rimane la questione educativa, poiché nella trasmissione di valori e di modalità sociali d'azione si forgiano le menti dei giovani e, in qualche misura, se ne determinano le scelte di vita, che potranno poi assumere una veste di continuità o di rottura rispetto ai modelli veicolati in famiglia. Da notare qui come questa trovi il suo asse nella figura materna che, pressoché ovunque, costituisce il primo riferimento per i figli rispetto al comprendere sia ciò che è bene e ciò che è male, sia come agire.

A partire dunque dal rapporto, coniugato al femminile, "educazione familiare- prevenzione del radicalismo giovanile" e basandomi sulle ricerche che conduco da una decina d'anni in Paesi quali il Mali, il Senegal e la Repubblica di Guinea, intendo procedere con un discorso a carattere antropologico, senza rivolgermi a un pubblico di lettori specializzati sulle realtà africane, ma con l'obiettivo di offrire qualche pista di confronto per chi opera, da noi, a livello pedagogico, in seno a realtà marcate dal meticciato culturale e dall'immigrazione.

Un chiarimento s'impone subito: nelle zone sahelo-sahariane, l'estremismo assume un volto per lo più religioso e riguarda un fondamentalismo islamico belligerante d'ispirazione wahhabita (o salafita), che assolda migliaia di ragazzi in nome di un ideale di fede intollerante, dove sono banditi il dubbio, l'interrogarsi e ogni valutazione di ordine personale, nel nome di un'adesione totalizzante a un messaggio che si qualifica (a torto) come portatore esclusivo della verità più alta, quella divina. Tale dottrina - negli Stati a cavallo del Sahara - non viene diffusa presso popolazioni dove l'islam è minoritario ma, al contrario, dove rappresenta la religione della maggioranza della gente ${ }^{2}$. Lappello rivolto ai seguaci del radicalismo violento non punta perciò a rivendicare i diritti di una credenza musulmana svilita da un tessuto collettivo che le è ostile o estraneo, ma a contestare un sistema politico nazionale giudicato ingiusto, al soldo di potenze straniere neocoloniali etichettate come blasfeme, il quale non assicura ai giovani un posto nella società e non offre loro una prospettiva accettabile di futuro ${ }^{3}$.

${ }^{2} \mathrm{Nel}$ quadro di questo articolo non è ovviamente possibile delineare in modo esaustivo la realtà geografica, culturale e soprattutto religiosa dell'immensa fascia sahelo-sahariana. Per una visione più puntuale e ad ampio spettro, si possono, fra gli altri, consultare: AA.VV. (2012a): Fronte del Sahara. Limes n. 5; AA.VV. (2012b): L'islam au-delà des catégories. Cahiers d'études africaines, nn. 206-207; AA.VV., (2020): Confluences aux extrêmes. Dossier. Afrique(s) en mouvement $\mathrm{n}$. 2; Robinson, 2004 (d'ora in poi, laddove non diversamente specificato, le note a piè di pagina si intendono a cura dell'Autrice, N.d.R.).

${ }^{3}$ È il caso, ad esempio, nella Nigeria nord-orientale, del gruppo jihadista Boko Haram. Il movimento è nato nel 2002 col nome di Jama'atu ahlus sunnah lidda'awati wal-jihad (Discepoli del Profeta per la propagazione dell' $i$ slam e la guerra santa). Denunciando la corruzione della classe politico-militare al potere, soggiogata da modelli 
$\mathrm{Al}$ proposito, commentando un'indagine sul campo, compiuta attraverso interviste approfondite rivolte a 800 giovani saheliani, il politologo Reda Benkirane parla di un islam combattente che - per reclutare meglio - esprime il «rigetto di un sistema che rigetta» $(2016$, pp. 23-45) . E aggiunge: "La radicalizzazione compie una rottura rispetto a un continuum storico, politico e culturale, ma è pure un modo di produrre (attecchendo) una nuova identità individuale [...], taglia certe radici per instaurarne altre, che si propagano poi in maniera reticolare ed in senso orizzontale» (Ibidem), circolando in forma virale.

\section{La donna e il processo educativo nelle società sahelo-sahariane}

Nella fascia africana che si estende dalle coste del Senegal, per toccare Paesi quali Gambia, Guinea-Bissau, Repubblica di Guinea, spingendosi poi nelle terre dell'interno lambite dal deserto (Mali, Burkina-Faso, Niger, Nigeria, Ciad), il ruolo che le consuetudini e i costumi sociali assegnano alla donna in seno alla famiglia coincide con la cura e l'educazione dei figli, in particolare durante la loro prima infanzia. Si tratta del compito connesso all'esigenza di tramandare fra le generazioni - con la parola, i gesti e la condotta - le conoscenze basilari tipiche delle diverse etnie, sorta di patrimonio immateriale che permette all'individuo, già in tenera età, di cominciare a inserirsi nel contesto collettivo di appartenenza.

Spiega al proposito Amadou Hampaté Ba, eminente studioso delle culture sahelo-sahariane:

La tradizione orale è una grande scuola di vita e tocca tutti gli ambiti. Può apparire persino caotica a quanti [dall'esterno, N.d.A.] non ne penetrano il segreto oppure può sconcertare lo spirito cartesiano abituato a separare la realtà in categorie rigorose. Nella tradizione, la dimensione spirituale e quella materiale non vengono mai scisse ma si passa senza cesure dall'esoterico all'essoterico, [grazie ad un discorso $o$ a pratiche, N.d.A.] che sanno mettersi alla portata di ciascuno, parlandogli secondo la sua capacità d'intendere e in funzione delle sue attitudini. La tradizione è al contempo religione, sapere, scienza della natura, apprendimento di un mestiere, storia, divertimento, ricreazione e ogni suo singolo dettaglio richiama un insieme unitario primordiale (1980, p. 193).

I valori alla base di tale bagaglio di conoscenze costituiscono l'oggetto di un lungo processo di formazione rivolto ai giovani, che le madri intraprendono ma che viene gestito, in una fase successiva, dal capo-famiglia (da intendere in senso lato: il padre, il nonno o lo zio paterno). Come osserva la storica Barbara M. Cooper, questi valori, in area sahelo-sahariana, sono espressi da concetti quali: «l'onore, il senso della vergogna, il pudore e il rispetto [...] sorta di marchio dell'umano e di un'educazione corretta, di cui è segno tangibile l'autocontrollo in presenza di coloro ai quali si deve deferenza» (2018, p. 74), ovvero gli adulti e, in primis, gli anziani della comunità. Si tratta di un modello d'insegnamento che tende, con gradualità e ricorrendo a percorsi rituali di tipo iniziatico, ad instillare presso i ragazzi e le ragazze il rispetto per le ge-

importati dall'Occidente, e qualificandosi come difensore delle comunità musulmane del Nord-Est, da sempre trascurate dal governo federale, il gruppo intende dar vita a una repubblica autonoma islamica, tesa ad applicare la sharia (normatività rivelata) nella versione "originaria". Il movimento si aggancia a un progetto collettivo, da mettere in esecuzione ricorrendo a una modalità insurrezionale. Sull'onda di tale filone battagliero, nel 2003, sono perpetrati i primi attentati, seguiti da atti di una violenza raccapricciante (uccisioni di civili, stupri, rapimenti fra cui, nel 2014, quello di 273 studentesse liceali). Nel frattempo, la Jamáatu assume la denominazione di Boko Haram. Questa espressione ha carattere polisemico e rimanda, in lingua haussa, a tre nozioni: il libro (dall'inglese book), lo stregone (boka) e la menzogna (boko), elementi che sono etichettati, in blocco, come proibiti (haram), quando frutto di un processo perverso di occidentalizzazione o di superstizione (cfr. Pelizzari, 2016, in particolare le pp. 83-84).

${ }^{4}$ D'ora in avanti, laddove non diversamente specificato, le traduzioni si intendono a cura dell'Autrice, N.d.R. 
rarchie che, nelle classi di età, trovano l'espressione più caratteristica. Ai principi tradizionali, si aggiungono però oggi - in un mondo nel quale alcune contrapposizioni (giovani/vecchi; minorenni/maggiorenni) risultano eccessivamente rigide o meno performanti sul piano collettivo - altri rapporti e gerarchie inedite che «si nutrono delle frustrazioni dei cadetti sociali o della manipolazione di queste» (Champy, Peatrick, 2016, pp. 116-117).

Alle nuove generazioni, specie se cresciute in un contesto urbano soggetto a influenze diverse, appare inaccettabile che sia ancora il capo-famiglia a decidere chi - fra i figli e i nipoti - proseguirà i suoi studi a livello liceale e universitario, con l'ambizione di entrare magari nell'amministrazione pubblica; chi dovrà invece dedicarsi al commercio, all'agricoltura o all'allevamento (a seconda del settore di attività privilegiato dalla famiglia); infine a chi spetterà il compito di salvaguardare la dimensione spirituale e la benevolenza divina sul gruppo parentale (per questo è usanza, in ambito musulmano, che almeno un bambino sia inviato, a partire dai quattro-cinque anni, alla scuola coranica con la prospettiva che un giorno diventi un marabutto, cioè un maestro e una guida per la comunità dei fedeli). I giovani faticano ad adeguarsi a "planning familiari" non liberamente vagliati, ma calati dall'alto e che puntano, per il successo, sulle figure dei maschi, mentre relegano le femmine a mera occasione per alleanze fra gruppi omologhi, concluse attraverso scambi matrimoniali (l'individuazione dello sposo, in un simile quadro, è naturalmente condizionata da esigenze che esulano dalla volontà personale delle ragazze).

Ho avuto riscontro di tali istanze nel corso di un dibattito, tenuto nel febbraio 2019 a Bamako, la capitale del Mali, sotto l'egida dell'Università di Scienze Giuridiche e Politiche (USJP). Intitolato Noi, tra forza costruttiva e strumento di dominio ed esclusione e moderato da un docente, l'appuntamento accademico ha visto la partecipazione vivace di oltre un centinaio di studenti, assai solleciti nel denunciare il peso per loro talvolta insostenibile di certe imposizioni provenienti dagli adulti. In una società ancora segnata nel profondo da consuetudini etniche e religiose considerate intoccabili, l'educazione impartita dalle famiglie rimane all'insegna dell'obbedienza nei riguardi delle persone più grandi, gli aînés (termine riferito a individui maggiori per età o statuto sociale). Ciò finisce per disegnare un rapporto intergenerazionale asimmetrico, dove il minore tace sempre e comunque, mentre il maggiore, dall'alto della sua supposta saggezza, acquisita con gli anni, pontifica a piacere.

Questo modello soffoca i giovani e dà loro la sensazione che, a casa, in patria, la loro voce non possa levarsi o sia destinata a rimanere inascoltata, prigioniera di canoni troppo austeri. Lo hanno dichiarato, senza giri di frase, durante il dibattito menzionato, due studentesse che, coraggiosamente, davanti ai compagni presenti nell'anfiteatro universitario, hanno ricordato quanto sia difficile affermarsi, per il genere femminile. Al di fuori di quanto prevedono le leggi dello Stato - il Mali è un Paese formalmente laico e la sua Costituzione, promulgata nel 1992, s'impegna a difendere i diritti della donna, senza discriminazioni di ordine sessuale (si vedano il Preambolo e il Titolo 1) - nel quotidiano, perdurano spinte insistenti affinché le ragazze, prima di aver completato il ciclo scolastico, si sposino, alleviando così i genitori dai costi del loro mantenimento e dalla responsabilità di garantirne la "purezza", la tanto vantata verginità prematrimoniale. Ma non basta, pratiche quali le mutilazioni genitali femminili e la poligamia perdurano, sponsorizzate o, quanto meno, non biasimate dai principali leader musulmani (l'islam sunnita è la fede del $90 \%$ dei cittadini maliani).

Complessità di questa natura - qui appena accennate - devono stimolare i ricercatori a leggere in tono più sfumato, attento alle specificità locali, il cammino dell'Africa saheliana contemporanea, inducendoli a distinguere meglio tra le diverse realtà e in particolar modo tra quanto capita in città e quanto capita nelle aree rurali, separando ambiti dove la modernità si afferma, pur fra mille contraddizioni, e contesti dove le usanze ataviche svolgono ancora una funzione preminente (Pelizzari, 2008, 2020). 
Nelle megalopoli sahelo-sahariane, in crescita continua e disordinata, abitudini di origine diversa finiscono per trovarsi inevitabilmente a confronto e, di conseguenza, s'insinua un pensiero meno uniforme, nel quale si evidenziano segni di apertura. Lo dimostra l'esempio delle due studentesse di Bamako intervenute all'incontro accademico: col loro prendere la parola, hanno disatteso i principi inneggiati da un modello educativo che celebra, come un valore, la sottomissione femminile, paziente e silenziosa. Le ragazze, nel contesto universitario, non sono però state scoraggiate ad esprimersi, al contrario, la loro voce è stata accolta come un segnale positivo (altrove, una simile performance sarebbe stata letta in termini d'impudicizia e sfrontatezza).

L'autonomia delle donne rispetto al decidere il proprio futuro e, almeno in parte, quello dei figli implica la messa in campo di pratiche inedite, estranee (o persino contrarie) alla mentalità veicolata dalla tradizione. Ciò può avvenire solo giocando sul livello d'istruzione acquisito, sulla mobilità sociale conseguibile nelle realtà urbane, sulle pressioni meno forti della famiglia allargata, sul contributo personale assicurato al budget coniugale attraverso un lavoro remunerato $^{5}$. Purtroppo, tale passaggio, che conduce le donne africane dall'assoggettamento a un ruolo da protagoniste, non ha luogo senza traumi o scontri. Lo ribadisce con ironia l'antropologo Kawélé Togola, scrivendo:

Quando, in una società patriarcale, l'evoluzione dei costumi avviene su iniziativa femminile - di chi, cioè, $[\ldots]$ non ha voce in capitolo ma deve subire, di chi è da sempre in uno stato di vassallaggio e di sottomissione all'uomo - emerge un problema. Quando, un progetto di società, dalla vocazione universale [quale l'affermarsi dei diritti umani in termini di uguaglianza di genere, N.d.A.], è promosso da attori dominati, le chances che sia accettato e ottenga l'adesione dei dominatori sono minime (2019, p. 160).

Eppure, la testimonianza di Fatoumata, giovane madre che ho incontrato a Bamako nel febbraio del 2020, attesta di un quadro in piena mutazione, dove le dinamiche collettive sono in fermento e la questione femminile compie passi importanti. Fatoumata ha svolto i suoi studi universitari in patria e in Algeria, per poi inserirsi a livello lavorativo in un'istituzione finanziaria internazionale della capitale maliana. Pur appartenendo a una famiglia musulmana e marabuttica, Fatoumata ha deciso di sposare un uomo cristiano e di lasciare che i suoi figli venissero cresciuti nella religione cattolica. Come lei stessa riconosce, tale scelta le è costata fatica e ostracismo da parte dei suoi ma, con gli anni, e soprattutto con la nascita dei bambini, le discordie si sono placate e lei ha ripreso i contatti con la propria parentela d'origine.

Fatoumata rivendica l'importanza, per le donne africane, d'imporsi in famiglia, di combattere per la loro emancipazione, nella certezza che "le cose possono cambiare, ma spetta alle donne volerlo". Sottolinea inoltre che, da tempo, "molte sorelle" (come le denomina) sono attive in ogni settore del Paese, dall'economia alla politica (le associazioni femminili chiedono persino che venga stabilita una "quota rosa", riservando il 30\% dei seggi parlamentari alle donne). Tale partecipazione agli affari pubblici ne legittima, a maggior ragione, il ruolo insostituibile nella sfera familiare, specie nei riguardi dell'educazione dei figli.

\footnotetext{
${ }^{5}$ Una serie d'interessanti spunti sull'evoluzione della famiglia in Africa è offerta dal volume L'Afrique des familles. La famille dans l'Afrique contemporaine entre changement et permanence, curato nel 2018 da Jean-Aimé Dibakana Mouanda e da Jean-Pierre Missié. Nel saggio collettaneo di taglio socio-antropologico, sono affrontate tematiche quali: le scelte matrimoniali (intese in termini di strategie personali attentamente studiate dagli attori sociali, soprattutto di sesso femminile, per migliorare il proprio status), il controllo della fecondità (dal rifiuto delle unioni precoci all'uso dei contraccettivi), i legami di parentela e i rischi che questi implicano (alla presupposta solidarietà di gruppo, sbandierata come un valore autoctono da difendere, si contrappongono, nella realtà, gelosie reciproche, forme di assoggettamento, timori connessi al ricorso a pratiche di stregoneria).
} 
Certo il caso di Fatoumata non corrisponde alla regola, ma non è neppure un'eccezione, se ci soffermiamo ad osservare l'Africa "delle metropoli", con la sua effervescenza e la molteplicità d'influenze che s'incrociano. Da ricordare, per altro, che circa il $40 \%$ della popolazione del continente vive oggi in città.

\section{L'islam nella regione sahelo-sahariana}

Per comprendere meglio le trasformazioni in atto rispetto al ruolo tradizionale della donna africana, con un accento specifico al quadro educativo familiare, è importante non sottostimare l'aspetto religioso, poiché tale dimensione ha un posto preponderante nell'esistenza collettiva, sia come fattore "collante" delle società sia, purtroppo, come fattore "disgregante" delle stesse. Lo dimostrano molte esperienze recenti, dove il contrasto fra le credenze assume toni partigiani e investe elementi identitari connessi all'appartenenza etnica.

È noto, ad esempio, come l'etnia peul (presente in vari stati dell'Africa dell'Ovest) venga vista da secoli come portatrice del messaggio islamico, che ha tentato di propagare ovunque persino ricorrendo alla "guerra santa"' . Oggigiorno, i suoi membri sono accusati di stretta connivenza con le milizie jihadiste del Sahel. Tali gravi sospetti (avvalorati da episodi sanguinosi) sono denunciati da gruppi quali i Dogon, un popolo di coltivatori che abita le regioni centro-orientali del Mali, dove tra mille contrasti tale etnia condivide il territorio con allevatori seminomadi peul. Antichi dissapori fra agricoltori e pastori, connessi ai diritti d'accesso a risorse scarse come l'acqua e le terre, riemergono adesso in chiave religiosa, contrapponendo, con qualche stortura, un islam pacifico di stampo mistico (sufi), a un islam estremista di stampo wahhabita.

Detta situazione - che incide tanto a livello politico quanto a livello socio-familiare rappresenta, sotto parecchi risvolti, un'eredità storica dell' islam sahelo-sahariano. Per questo, può essere utile delinearne, seppure schematicamente, il profilo. La fede musulmana è presente nell'area dall'XI secolo e la sua diffusione ha seguito le vie dei traffici commerciali attraverso il deserto. All'epoca, le carovane trasportavano l'oro dalle regioni degli antichi imperi del Wagadou e del Mali, in cambio di manufatti provenienti dal Maghreb.

In concomitanza, si sono formati, nei luoghi di scambio, dapprima, piccoli insediamenti di mercanti arabi islamizzati, poi tale fede si è trasformata in uno strumento di affermazione e di dominio per le élites locali. In una terza fase, fra il XVIII e il XIX secolo, il messaggio maomettano si è diffuso anche presso i ceti popolari, sino a rimpiazzare, almeno in superficie, le radici animiste. Le conversioni legate a questa terza fase sono avvenute essenzialmente ad opera dei leader delle confraternite mistiche sufi, che non hanno disdegnato lo strumento del jihad, come nel caso del califfato di Sokoto (costituito nel Nord-Est della Nigeria attuale da Usman Dan Fodjo, maestro spirituale e stratega militare di etnia peul/ fulani) oppure dell'impero peull

\footnotetext{
${ }^{6} \mathrm{Nel}$ romanzo intitolato Peuls (2004), sorta di saga familiare che si estende su un lunghissimo periodo e tocca molte regioni della fascia sahelo-sahariana, lo scrittore guineano Tierno Monénembo ricostruisce i miti e la storia di questa peculiare etnia (denominata anche, a seconda dei luoghi, fuldé, fulani, pular, toukouleur ecc.). L'Autore insiste, da un canto, sulla posizione sociale dei nomadi peul, collocati spesso ai margini delle civiltà contadine stanziali e, dall'alto, sul loro essersi ancorati al fattore religioso, con una conversione all'islam quasi generalizzata e già attestata nel XVI secolo. La fede maomettana diviene rapidamente, per i pastori peul, elemento d'identità e di stimolo solidale, fonte di orgoglio, di rivalsa e, persino, di affermazione politico-militare. Sono infatti protagonisti, sotto l'egida di capi carismatici che giocano un doppio ruolo, religioso e militare, di una serie di jihad condotte fra il XVIII e il XIX secolo in una vastissima area, corrispondente oggigiorno a Stati quali Senegal, Repubblica di Guinea, Nord-Est della Nigeria, Mali e Niger. Sui costumi tipici dei Peul e sul modello educativo che, in parte, tramandano ancora alle nuove generazioni in epoca attuale (Botta Somparé, 2015).
} 
toukouleur fondato da un altro predicatore combattente, Al-Haji Umar Tall, nell'alta valle del fiume Niger (Mali attuale).

Oggigiorno, un terzo degli abitanti dell'Africa è di religione musulmana (sunnita). L' $i$ slam si caratterizza qui per la sua contaminazione con le culture autoctone e per il prestigio delle confraternite sufi (Otayek, Soares, 2009). Negli ultimi decenni, però, il predominio di queste è stato contestato dai movimenti salafiti (dall'arabo al-salaf al-salih = i pii antenati, cioè i compagni del Profeta Maometto), conosciuti anche come movimenti wahhabiti (dal nome del teologo Abd al-Wahhab vissuto in Arabia nel XVIII secolo, propugnatore di un ritorno alla purezza originaria della fede attraverso il concetto di “tawhid = unicità di Dio" e l'interpretazione letterale del Corano).

I movimenti salafiti/wahhabiti rivendicano la necessità d'islamizzare la società in ogni suo aspetto, in quanto solo in un quadro del genere i fedeli possono vivere a pieno la loro credenza. Ciò implica l'applicazione della sharia o "normatività rivelata". Ricordiamo che la sharia non corrisponde a un insieme di leggi omogenee ma ha statuto ideale: creare in terra un regno adeguato al volere divino.

L' islam delle confraternite mistiche sufi si caratterizza invece per il culto dei santi fondatori (shaykh), per una liturgia che prevede, oltre le preghiere canoniche, la ripetizione cantilenante del nome di Dio (dhikr) e, infine, per il sincretismo con gli usi ancestrali (quali la pratica dell'infibulazione femminile, il ricorso a sacrifici o alla divinazione ecc.). I capi delle confraternite, i marabutti, detengono un potere carismatico e sono custodi della benedizione divina (baraka) che trasmettono ai loro accoliti. I marabutti gestiscono inoltre le scuole coraniche, centri d'insegnamento delle regole di base dell'islam e di studio della teologia. In alcuni casi, meno rari nelle grandi città dell'Africa occidentale, le confraternite possono anche essere dirette da donne, che assumono allora sia una funzione di guide spirituali, connessa alle loro competenze teologiche, magari apprese (ereditate) nel seno alla famiglia, sia una funzione a carattere più esoterico e magico-terapeutico ${ }^{7}$.

\section{L'apporto femminile quale baluardo rispetto all'estremismo}

A partire da quanto indicato, risulta più agevole comprendere come l' islam sahelo-sahariano (tanto nelle vesti più tradizionali del sufismo quanto in quelle più recenti del wahhabismo) abbia plasmato e continui a plasmare, in modo viscerale, la vita delle comunità: fede e appartenenza politico-culturale si fondono in maniera indelebile nella costruzione dell'identità personale.

Ciò vale anche per le donne, rispetto al loro compito di madri ed educatrici. Di conseguenza, fronte all'irrompere del radicalismo islamico combattente di matrice wahhabita (ribadiamo comunque che il wahhabismo non coincide necessariamente col jihad e che la sua componente preponderante rifiuta il ricorso alla violenza), l'apporto femminile quale baluardo contro l'estremismo risulta prezioso.

\footnotetext{
${ }^{7}$ Scrive l'antropologa Amber Gemmeke: in varie parti «dell'Africa occidentale, dagli anni 1970, l'accesso femminile all'educazione islamica ha permesso a un crescente numero di donne di affermarsi come predicatrici e teologhe, disposte a sfidare la divisione patriarcale dei ruoli. Anche in Senegal, l'affermarsi delle donne come autorità musulmane in ambito mistico è in aumento, sebbene in modo meno appariscente. Le aree urbane, e specialmente Dakar, sembrano offrire alle donne opportunità inedite di carriera come marabutte, fenomeno che non ha equivalente nelle aree rurali. Dakar, con la sua massa di persone alla ricerca di un lavoro, di una casa, di uno statuto matrimoniale legittimo, che si muovono nell'ambito di un mercato fortemente competitivo, sembra costituire un terreno assai fertile per pratiche esoteriche inedite e per nuove forme di potere al femminile» (2009, p. 147).
} 
Da una parte, le donne possono essere le porta-voci di un messaggio religioso legato alle consuetudini locali, dunque prossimo al proselitismo delle confraternite sufi. Queste ultime osteggino apertamente le posizioni integraliste e radicali dell'islamismo combattente, per difendere invece una pratica della fede più tollerante, capace di fondersi con le culture del luogo. In effetti, una vivace concorrenza contrappone ovunque, ma soprattutto nelle realtà urbane, la prospettiva dell' islam sufi e quella dell' islam wahhabita, considerata dai più come estranea all'Africa e sottomessa all'influenza delle monarchie della Penisola arabica.

D'altra parte, le donne - specialmente se istruite ed emancipate - possono trovare nel messaggio wahhabita non violento (o pietista, come viene definito sul posto) una via per affermare una pratica di fede più pura e meno ancorata alla pesante influenza dei marabutti, dunque un modo di vivere, nel quotidiano, la propria credenza a tutto tondo, senza compromessi con la tradizione e i costumi ancestrali. L'idea sottostante è che «il/la buon/a musulmano/a" sia portatore/portatrice di un progetto di società da realizzare tanto nel quadro familiare come in quello collettivo, senza cedere ai compromessi, ma adottando strategie "fluide" e personalizzate, quasi tagliate su misura» (Leblanc, Gomez-Perez, 2007, pp. 39-59).

Come mi ha spiegato Mame, una giovane giornalista di Dakar in occasione dei nostri incontri nella capitale senegalese avvenuti nel novembre 2019, l'obiettivo delle madri africane nei nostri tempi turbolenti dovrebbe essere - secondo le sue stesse parole - di «offrire dei punti di riferimento ai figli e d'indicare loro una direzione da prendere nella vita». Senza tale supporto il rischio di derive per le nuove generazioni è enorme, confrontate come sono alle difficoltà, sul piano dell'occupazione e dello sviluppo, tipiche di Paesi che stanno conoscendo una crescita demografica troppo rapida e mal gestita dai governi nazionali. Secondo Mame, i movimenti radicali violenti, con la loro proposta di lottare nel nome della fede, allettano una gioventù «en mal de futun - ovvero, priva di prospettive - e, di fatto, si sostituiscono alla famiglia e allo stato, dimostratisi incapaci di dare una speranza concreta a tanti ragazzi. Solo una risposta in termini concreti di sviluppo potrebbe arginare una deriva che, pur rivestendo un'apparenza religiosa e jihadista, cela il bisogno pressante e ineludibile delle nuove generazioni di costruirsi, costi quello che costi, un domani.

Nel concludere il suo discorso, Mame cita poi un noto proverbio: «se un figlio, diventato adulto, ha successo nella vita, l'intero villaggio se ne vanta, quando invece un giovane fallisce, è incolpata la sola madre dello smacco». La tradizione rivela così il carattere essenziale dell'educazione impartita in casa dalle madri e, in una certa misura, sposta l'asse centrale della famiglia patriarcale dall'uomo alla donna, caricando sulle spalle di quest'ultima il fardello più pesante: la costruzione di un futuro prospero per la società.

Insomma, sebbene - per molti versi - si possa parlare di una certa invisibilità delle donne africane nella storia recente della lotta alla violenza di matrice radicale e fondamentalista, ovvero di una loro apparente assenza in veste di attrici di primo piano nella prevenzione e nel contrasto dell'estremismo jihadista, la situazione sul campo risulta assai più sfaccettata e dinamica. Il loro ruolo, meno appariscente, sembra giocarsi dietro le quinte o, meglio, in un quadro domestico raramente percettibile agli occhi degli analisti frettolosi.

\section{Riferimenti bibliografici}

AA.VV. (2012a): Fronte del Sahara. Limes. Rivista italiana di geopolitica, n. 5, pp. 7-236.

AA.VV. (2012b): L'islam au-delà des catégories. Cahiers d'études africaines, nn. 206-207, pp. 329-686.

AA.VV. (2020): Confluences aux extrêmes. Dossier: Extrémisme violent au féminin: un mélange des genres? Afrique(s) en mouvement, n. 2, pp. 6-102.

Hampaté Ba A. (1980): La tradition vivante. In J. Ki-Zerbo (dir.): Histoire générale de l'Afrique. Tome I. Paris: Unesco, pp. 191-230. 
Benkirane R. (2016): Radicalisation, violence et (in)sécurité. Ce que disent 800 Sahéliens. Centre pour le dialogue humanitaire: UNDP-PNUD (https://radical.hypotheses.org/files/2017/06/I_Etude_PNUDHD_perceptions_Sahel_Resume_ex\%C3\%A9cutif.pdf; data di ultima consultazione: 10.11.21).

Biagioli R., Gonzáles-Monteagudo J., Petruzzi C. (2018): Ruolo e formazione degli educatori. Pedagogia e metodologie per le comunità di accoglienza dei minori stranieri. Torino: L'Harmattan Italia.

Botta Somparé E. (2015): Éducation familiale et scolaire dans une société pastorale guinéenne. Paris: L'Harmattan.

Champy M., Peatrick A.M. (2016): Pour une anthropologie critique de la jeunesse en Afrique. Afrique contemporaine, 259(3), pp. 116-117.

Cooper B.M. (201 8): Regard historique sur la honte et les pratiques reproductives au Soudan central. In C. Baroin, B.M. Cooper (dir.): La honte au Sahel. Pudeur, respect, morale quotidienne. Paris: L'Harmattan/Sépia, pp. 51-100.

Dibakana Mouanda J.A., Missié J.P. (dir.) (2018): L'Afrique des familles. La famille dans l'Afrique contemporaine entre changement et permanence. Paris: L'Harmattan.

Gemmeke A. (2009): Marabout Women in Dakar: Authority, Creativity and Islamic Knowledge. Africa. Journal of the International African Institute, 79(1), pp. 128-147.

Leblanc M.N., Gomez-Perez M. (2007): Jeunes musulmans et citoyenneté culturelle: retour sur des expériences de recherche en Afrique de l'Ouest francophone. Sociologie et Societés, 39(2), pp. 39-59.

Monénembo T. (2004): Peuls. Paris: Seuil.

Otayek R., Soares B. (dir.) (2009): Islam, état et société en Afrique. Paris: Karthala.

Pelizzari E. (dir.) (2020): Éducation scolaire, droits humains, läcité: quelle place pour la jeunesse en Afrique de l'Ouest? Analyses socio-anthropologiques à partir du cas du Mali. Paris/Torino: L'Harmattan/L'Harmattan Italia.

Pelizzari E. (2016): Il sacrificio alle radici della logica religiosa. Una lettura dell'islam in Africa occidentale. In M.L. Maniscalco, E. Pelizzari (a cura di): Deliri culturali. Sette, fondamentalismi religiosi, pratiche sacrificali, genocidi. Torino: L’Harmattan Italia, pp. 60-90.

Pelizzari E. (a cura di) (2008): Partecipazione politica e costruzione sociale: l'Africa al femminile (studi di genere). Inchiesta, n. 161 (dossier). Bari: Dedalo.

Robinson D. (2004): Muslim Societies in African History. New York: Cambridge University Press.

Silva C.(2004): Comunicazione, relazione, mediazione e dialogo tra culture diverse. In L. Borghi, C. Barbarulli (a cura di): Figure della complessità. Genere e intercultura. Cagliari: CuEC, pp. 173-184.

Silva C. (2017): Cape Verdean Immigrant Women in Italy. Pedagogia oggi, 15(1), pp. 373-383.

Togola K. (2019): Les dynamiques de genre en Afrique et au Mali. Paris: L'Harmattan. 
